\title{
THE
}

\section{Predators, Prey Refuges, and the Spatial Scaling of Density- Dependent Prey Mortality}

\author{
Graham E. Forrester \\ University of Rhode Island, gforrester@uri.edu \\ Mark A. Steele
}

Follow this and additional works at: https://digitalcommons.uri.edu/nrs_facpubs

Terms of Use

All rights reserved under copyright.

\section{Citation/Publisher Attribution}

Forrester, G. E. \& Steele, M. A. (2004). PREDATORS, PREY REFUGES, AND THE SPATIAL SCALING OF DENSITY-DEPENDENT PREY MORTALITY. Ecology, 85, 1332-1342. doi: 10.1890/03-0184

Available at: http://dx.doi.org/10.1890/03-0184

This Article is brought to you for free and open access by the Natural Resources Science at DigitalCommons@URI. It has been accepted for inclusion in Natural Resources Science Faculty Publications by an authorized administrator of DigitalCommons@URI. For more information, please contact digitalcommons-group@uri.edu. 


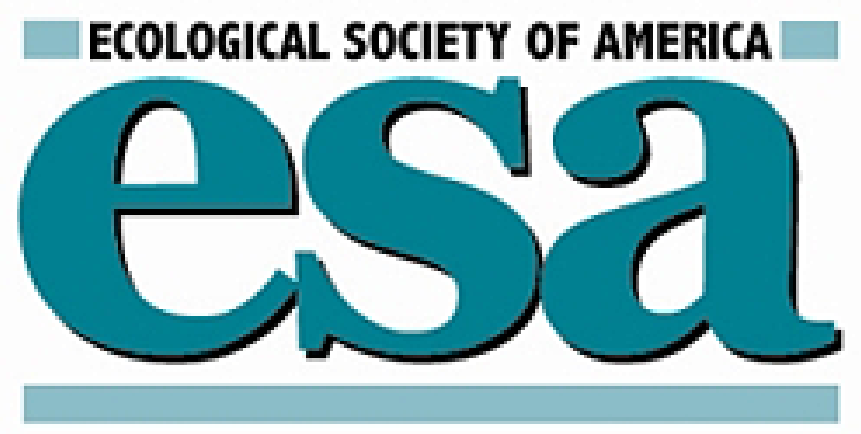

Predators, Prey Refuges, and the Spatial Scaling of Density-Dependent Prey Mortality Author(s): Graham E. Forrester and Mark A. Steele

Source: Ecology, Vol. 85, No. 5 (May, 2004), pp. 1332-1342

Published by: Ecological Society of America

Stable URL: http://www.jstor.org/stable/3450175

Accessed: 21/03/2013 12:20

Your use of the JSTOR archive indicates your acceptance of the Terms \& Conditions of Use, available at http://www.jstor.org/page/info/about/policies/terms.jsp

JSTOR is a not-for-profit service that helps scholars, researchers, and students discover, use, and build upon a wide range of content in a trusted digital archive. We use information technology and tools to increase productivity and facilitate new forms of scholarship. For more information about JSTOR, please contact support@jstor.org. 


\title{
PREDATORS, PREY REFUGES, AND THE SPATIAL SCALING OF DENSITY-DEPENDENT PREY MORTALITY
}

\author{
Graham E. Forrester ${ }^{1,3}$ AND MARK A. SteElE ${ }^{2}$ \\ ${ }^{1}$ Department of Natural Resources Science, University of Rhode Island, Kingston, Rhode Island 02881 USA \\ ${ }^{2}$ Marine Science Institute, University of California, Santa Barbara, California 93106 USA
}

\begin{abstract}
We tested the biological cause of density-dependent mortality in the bridled goby (Coryphopterus glaucofraenum), a small coral reef fish, and evaluated whether this knowledge allowed us to detect density dependence at different spatial scales in natural habitats. To identify the biological cause of density dependence, we manipulated both population density and the availability of shelter (crevices used as refuges from predators) in small plots of continuous reef. We detected strong density-dependent mortality in plots with few refuges, but mortality was density independent in plots with abundant refuges, indicating that limited shelter causes density dependence. Predator density was unrelated to the density of gobies and refuges, suggesting that predators displayed a type III functional response in patches with few refuges. In a second experiment, we manipulated goby density within replicate plots of three sizes $\left(4,16\right.$, and $\left.64 \mathrm{~m}^{2}\right)$ that varied naturally in the availability of refuges. If refuge availability was ignored, mortality appeared to be density independent at all scales. If, however, plots were grouped by refuge availability, mortality was density dependent in plots with few refuges, but low and density independent in plots with many refuges at all spatial scales. Understanding the mechanism of density dependence (refuge shortage) was thus required to measure the strength of density dependence in natural, spatially variable, habitat. We suggest that density dependence was detectable in plots of different sizes because the relationships between the densities of gobies, refuges, and goby predators were similar across the spatial scales we studied. Our work demonstrates that identifying the biological interactions that cause density dependence, and characterizing the spatial domains at which those interactions operate, will be important to accurately assess the effects of density dependence on population dynamics.
\end{abstract}

Key words: coral reefs; Coryphopterus glaucofraenum; density dependence; density-dependent mortality in reef fishes; Gobiidae; population regulation; prey refuges and predation; reef fishes; shelter limitation; spatial scale.

\section{INTRODUCTION}

Density-dependent interactions can have a strong influence on population dynamics and on the long-term stability of populations, yet identifying the mechanisms of density dependence and determining the extent to which they drive population dynamics remains a major challenge in population ecology (Auerbach et al. 1995, Begon et al. 1996). Experimental manipulation of population density is considered the most reliable method to define density-dependent interactions (Harrison and Cappuccino 1995). Whereas experimental tests of density dependence are rare in many systems (Harrison and Cappuccino 1995), most tests for density dependence in populations of reef fishes have used this method (Hixon and Webster 2002). As is true generally, some of these experiments detect intense density-dependent mortality, whereas others find it to be weak or absent (reviews by Hixon and Webster 2002, Osenberg et al. 2002). The variable outcome of these experiments

Manuscript received 17 March 2003; revised 16 September 2003; accepted 17 September 2003. Corresponding Editor: P. T. Raimondi.

${ }^{3}$ E-mail: gforrester@uri.edu on reef fishes has engendered controversy over the actual strength of density-dependent mortality and how it affects population dynamics in reef fishes (reviews by Doherty 1991, Jones 1991, Caley et al. 1996, Doherty 2002, Hixon and Webster 2002, Osenberg et al. 2002). Here we address three interrelated issues that affect our ability to reliably assess the intensity of density-dependent interactions using field manipulations of population density: the underlying biological cause of density dependence, spatial heterogeneity in features of the habitat, and the spatial extent of the manipulation. We examine how these factors affect the outcome of experimental tests for density dependence in an attempt to resolve some of the controversy surrounding the influence of density-dependent interactions.

Density-dependent mortality can be caused by resource limitation, certain forms of predation, disease, or parasitism. For organisms like reef fishes, for which causes of death cannot be readily determined by observation, identifying these biological causes of density-dependent mortality is difficult (Cappuccino 1992, Crawley 1992). In such cases, a powerful method to overcome this difficulty is to manipulate both density and the putative cause of 
density dependence in a cross-factored experiment. The rationale is that if a specific agent of mortality is causing density dependence, eliminating or augmenting it experimentally should eliminate or augment density dependence. A few such experiments have been performed on reef fishes, and though the cause of density dependence was not always established, predation and interspecific competition have been identified as agents of density-dependent mortality in some species (Forrester 1990, Hixon and Carr 1997, Forrester and Steele 2000, Carr et al. 2002, Holbrook and Schmitt 2002).

In this study, we test a potentially widespread cause of density-dependent mortality: density-dependent predation due to a shortage of structural refuges used by prey (Lynch et al. 1998 and references therein). Like many animals, most reef fishes use structural habitat features for shelter (Helfman 1993), and their abundance is often highest at sites that provide abundant shelter because these sites are either structurally complex or contain many holes/crevices (Caley and St John 1996, Ault and Johnson 1998, Holbrook et al. 2000, and references therein). Predators often account for a large fraction of mortality (e.g. Carr and Hixon 1995, Connell 1997, Hixon and Carr 1997, Steele 1997, Forrester and Steele 2000, Steele and Forrester 2002) and predator impacts can be reduced by habitat manipulations that increase the availability of shelter for prey or reduce prey encounter rates with predators (Steele 1999, Anderson 2001, and references therein). Progressively diminishing access to refuges as prey become more crowded should increase their vulnerability to predation and so cause density-dependent prey mortality (Beukers and Jones 1998). There is, however, little direct evidence to support the hypothesis that limited shelter causes density-dependent prey mortality in any natural system (but see Holbrook and Schmitt 2002). We tested this hypothesis directly by manipulating population density and refuge abundance in a factorial experiment.

Like most ecological experiments (Karieva and Anderson 1986), manipulations of reef fish density have been done on habitat patches no more than a few meters in extent (reviews by Jones 1991, Caley et al. 1996, Hixon and Webster 2002). In addition to their small size, these habitat patches were also physically isolated from other patches by inhospitable habitat. Both the size of study plot (e.g., Force and Moriarty 1988, Freeman and Smith 1990, Rothman and Darling 1990, Hopper et al. 1991, Ray and Hastings 1996) and whether the areas are isolated fragments or embedded within continuous habitat (Stewart and Jones 2001), can affect the outcome of tests for density dependence. Whether the strength of density dependence changes with spatial scale is likely to depend on which biological interactions cause density dependence (Kawat 1997). For example, spatially density-dependent predation caused by predator aggregation (Murdoch and Oaten 1975) only occurs at spatial scales across which searching predators respond to prey density (Rothman and Darling 1990, Whitlatch et al. 1997). Changes in the strength of other density-dependent interactions with spatial scale have been little studied. We therefore tested whether the strength of density dependence due to limited shelter was affected by the spatial extent (Wiens 1989) of our experiments. We tested for effects of spatial extent simply by performing density manipulations in plots of different size (Bertolo et al. 1999 and references therein).

\section{Objectives}

We present the results of two field experiments done on plots within larger continuous reefs. We chose a study species that has consistently displayed densitydependent mortality on small isolated patch reefs (Forrester 1995, Forrester and Steele 2000) so that we could evaluate the influence of habitat configuration (isolated patch reefs vs. continuous reef) on density dependence. In the first experiment, we tested whether density dependence is caused by a shortage of refuges from predation by manipulating both prey density and the abundance of refuges within small plots. In the second experiment, we tested whether the strength of density dependence was affected by spatial scale by manipulating density within plots of varying size. Since the plots used in the second experiment also varied naturally in refuge abundance, we tested for an effect of refuge abundance on the strength of density dependence in addition to testing the effect of plot size. In combination, these experiments allow us to test whether understanding the mechanism of density dependence (shelter limitation) permits us to accurately assess its effect in spatially heterogeneous habitats of varying extent.

\section{Methods}

Study species and sites

Our study species, Coryphopterus glaucofraenum Gill (the bridled goby), occurs on coral reefs throughout the Caribbean (see Plate 1). Like most reef fishes, bridled gobies have planktonic larvae, and larvae settle to reefs at $6.5-8.0 \mathrm{~mm}$ SL (Sponaugle and Cowen 1994). Gobies mature at $22-25 \mathrm{~mm} \mathrm{SL}$ and reach $\approx 55$ $\mathrm{mm} \mathrm{SL}$, but rarely live longer than a year (M. A. Steele and G. E. Forrester, unpublished data). We worked on adult gobies $(>25 \mathrm{~mm}$ standard length [SL]), which maintain home ranges up to a few square meters in area. Neighboring home ranges often overlap, and aggression between neighboring adults is common. A mix of sand and hard substratum (low-relief coral or rubble) within the home range is important because gobies feed on sand-dwelling meiofauna, but use crevices at the reef/sand interface as refuges from predators. Gobies are cryptic when on sand, which may allow them to feed and avoid detection by some predators. When 


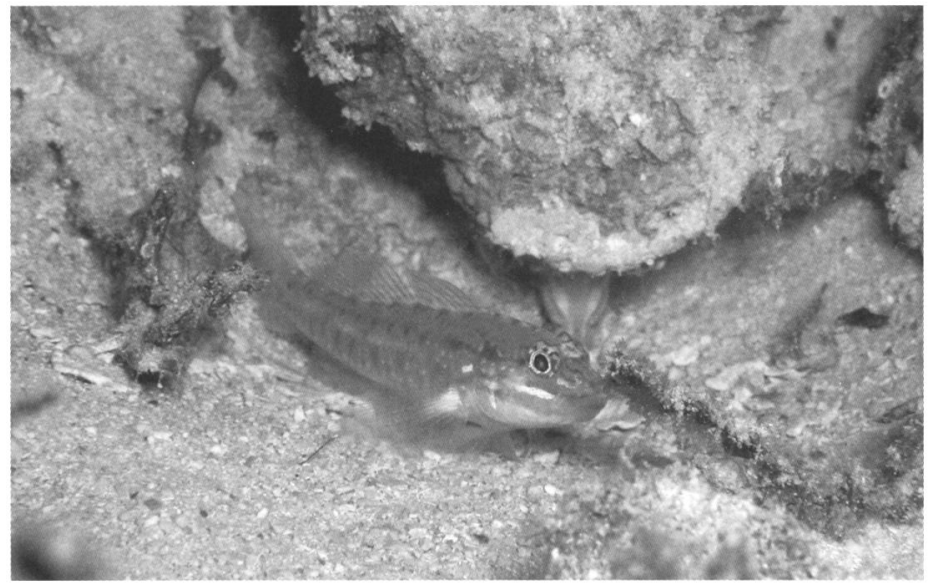

Plate 1. A bridled goby (Coryphopterus glaucofraenum) waits near the entrance to a crevice. When gobies are threatened or attacked by large predatory fishes they flee to small crevices at the reef/sand interface like this one. Photo credit: Mark Steele.

threatened or attacked by predators, mainly larger piscivorous fishes, gobies retreat temporarily to a refuge. A home range usually contains more than one refuge, and, since home ranges overlap, more than one goby may use any given refuge. Bridled gobies commonly occupy two habitat types: small patch reefs, where they reside at the sand/reef interface, and larger continuous habitat where coral/rubble is interspersed with enough sandy area for feeding. The cause of density-dependent mortality in gobies was unknown, but since predation is a major source of goby mortality (Forrester and Steele 2000, Steele and Forrester 2002) and gobies use structural refuges to escape predators, we hypothesized that a shortage of refuges might cause density dependence.

We worked near Guana Island, British Virgin Islands $\left(18^{\circ} 29^{\prime} \mathrm{N}, 64^{\circ} 35^{\prime} \mathrm{W}\right)$ and near Lee Stocking Island, Bahamas $\left(23^{\circ} 46^{\prime} \mathrm{N}, 76^{\circ} 10^{\prime} \mathrm{W}\right)$. Both study sites were large (thousands of square meters) expanses of continuous goby habitat, composed of sand interspersed with low-lying reef and coral rubble. Sites were all in shallow water $(3-10 \mathrm{~m})$ and in locations sheltered from waves. Adult densities up to 6 gobies $/ \mathrm{m}^{2}$ are regularly recorded in censuses of plots $\left(2 \mathrm{~m}^{2}\right)$ at our study sites, although densities up to 15 gobies $/ \mathrm{m}^{2}$ are occasionally observed (Forrester 1995, Forrester and Steele 2000; G. E. Forrester and M. A. Steele, unpublished data). The main confirmed predators of adult gobies at our sites are all generalist piscivorous fishes: lizardfish (Synodus spp.), graysbys (Epinephelus cruentatus), red hinds (E. guttatus), coneys (E. fulvus), hamlets (Hypoplectrus spp.), and trumpetfish (Aulostomus maculatus).

\section{Quantifying refuge use in bridled gobies}

Defining which crevices gobies consider a suitable refuge is difficult, but based on our observations of bridled gobies and the sorts of crevices to which they flee when threatened ( $>800$ SCUBA hours per author), suitable crevices are usually at the junction between sand (patches roughly $0.4 \mathrm{~m}^{2}$ in area or larger) and some hard substratum ( $>2 \mathrm{~cm}$ high and $5 \mathrm{~cm}$ wide). To corroborate our impressions about the size of crevices used as refuges, we measured all crevices at the sand-reef interface in a $6 \times 6 \mathrm{~m}$ plot of goby habitat near Guana Island. Forty-five of the 195 crevices measured were being used as refuges when surveyed (gobies fled there when disturbed by our approach). Occupied crevices had depths, heights and widths between 3 and 50, 2 and 16, and 3 and $55 \mathrm{~cm}$, respectively. To compare our impressions of what constitutes a refuge, both authors counted refuges within $164 \times 4$ m plots near Lee Stocking Island. Our refuge counts were well correlated $(r=0.86, P<0.001)$, suggesting that we could make rough estimates of the actual number of refuges available.

To describe the natural distribution of refuges, we counted refuges in 201 plots near Guana Island $(2 \times$ $2 \mathrm{~m})$ and in 80 plots near Lee Stocking Island $(4 \times 4$ $\mathrm{m})$. To assess the range of refuge densities that represented low, medium, and high levels at our sites, we divided the frequency distribution into three equal parts (terciles). The estimated refuge densities (refuges $/ \mathrm{m}^{2}$ ) for each tercile were as follows: low $=0.25-2.75$ refuges $/ \mathrm{m}^{2}$, medium $=2.75-4.25$ refuges $/ \mathrm{m}^{2}$, high $=$ $4.25-10.25$ refuges $/ \mathrm{m}^{2}$. To assess whether our estimates of refuge density were correlated with more general measures of habitat "complexity," we also measured rugosity (Rogers et al. 1982) and the percentage cover of coral, rock, and rubble in these plots. The percentage cover of hard substratum was correlated with the number of crevices (Guana Island: $n=201, r=0.81, P$ $<0.001$, Lee Stocking Island: $n=80, r=0.82, P<$ 0.001 ), so we used this as a rough index of refuge density in Experiment 2. Because many of the crevices gobies use as refuges are at the junction between sand and low-lying rock/rubble, refuge density was not correlated with rugosity $(n=80, r=-0.08, P=1.00)$, and only weakly with percentage cover of live coral $(n$ $=80, r=0.28, P=0.013)$. 


\section{Experiment 1: Is density dependence caused by limited shelter?}

To test whether shelter limitation caused density dependence, we used an analysis of covariance (ANCOVA) design. We manipulated both goby density (a covariate) and the density of refuges they use to escape predation (a categorical variable) within plots. Since we viewed our counts of refuge density as rough estimates, we treated refuge density as a categorical variable with three levels: low $\left(0.4-3.4\right.$ crevices $\left./ \mathrm{m}^{2}\right)$, medium (3.4-5.0 crevices $\left./ \mathrm{m}^{2}\right)$ and high (5.0-9.7 crevices/ $\mathrm{m}^{2}$ ). The levels of refuge density correspond roughly to the natural distribution of refuges at our sites (see Quantifying refuge use in bridled gobies). There were six replicate plots per refuge treatment, and within each refuge treatment a different density of gobies was established on each plot. Goby densities spanned the natural range at our sites.

Our main response variable was goby mortality, though we also measured emigration and some behaviors. Our primary aim was to test for an interaction between the effect of goby density and refuge abundance, because if density dependence in bridled gobies is caused by shortage of refuges, then density dependence should be reduced or eliminated when access to refuges is increased. This would be indicated in the ANCOVA by a significant interaction term due to a reduced slope of the relationship between mortality and density where refuges are common.

This experiment was conducted on the leeward, south side of Guana Island. Experimental plots were 4 $\times 4 \mathrm{~m}$ areas of goby habitat that were marked at the corners and separated from other plots by 4-6 m. Divers first estimated the percent cover of coral/rubble on each plot as a rough guide to the number of goby refuges present. Then, coral and rubble were added to 9 of the 18 plots in order to increase the availability of goby refuges. Divers arranged pieces of coral and rubble (15-40 cm long) within the plots, so as to maximize suitable shelter for the gobies. Coral/rubble was also added to the 9 nonaddition plots and then immediately removed to control for the disturbance of refuge addition. After the addition, divers counted the final number of suitable goby refuges on each plot.

To complete the manipulation, we adjusted the density of gobies ( $>25 \mathrm{~mm} \mathrm{SL}$ ) on each plot to the desired level. It was not logistically feasible to remove all resident gobies on the plots and replace them with individuals transplanted from elsewhere. Instead, some resident fish were left in each plot and formed part of the experimental population. When the natural density of residents was above the desired density, we removed some residents from the plot and then added gobies captured elsewhere to the plots to bring densities up to the desired level. If the natural goby density on a plot was below the required level, we simply transplanted additional fish from elsewhere. All plots were thus ultimately populated by a mix of resident and transplanted gobies $(33-61 \%$ of fish on a plot were residents). Lack of familiarity with the plot might cause transplants to access shelter less effectively or be more vulnerable to predators than established residents. We therefore performed separate tests for treatment effects on residents and transplants to check this possibility.

All adult gobies on the plots were tagged before the start of the experiment so individuals could be recognized. Small, uniquely coded $(1 \times 2.5 \mathrm{~mm})$ plastic Visual Implant Tags (NorthWest Marine Technology, Incorporated, Shaw Island, Washington, USA) were injected under the fishes' skin and could be viewed underwater by divers without capturing the fish. Prior work shows that these tags have no detectable adverse effect on gobies and do not increase their conspicuousness to predators (Malone et al. 1999; G. E. Forrester, unpublished data). Fish transplanted to the experimental plots were captured by divers using hand nets and anesthetic $100-150 \mathrm{~m}$ from the experimental site. Divers injected a tag into each fish immediately after capture, and tagged gobies were kept underwater in plastic bags for 20-120 minutes before being swum to the appropriate experimental plot and released. Residents were simply captured from their plot, tagged, and then released immediately at the point of capture.

It took 2-4 days to complete the process of tagging and transplanting fish onto a plot. The experiment was started 2-3 days later, to ensure that transplants had established themselves on the plots. Because the process was labor intensive, the start date differed among plots and was staggered over nine days (25 June-3 July 2000). Once the experiment began, divers censused the plots at intervals. Detailed censuses were made early in the experiment (on days 9-13) and at its end (days 49-52). Other tag-recapture studies in similar habitat using $\approx 4000$ gobies show that individuals rarely relocate their home range, and if so, rarely move $>5 \mathrm{~m}$ (M. A. Steele and G. E. Forrester, unpublished data). To check for emigrants, we thus carefully searched the area within $10 \mathrm{~m}$ of each plot and searched the area within $10-25 \mathrm{~m}$ of the plots less carefully. We noted the location of all tagged individuals on and off the plots, and also estimated the size and location of any untagged adult gobies that immigrated to the plots. The location of gobies was recorded $( \pm 0.2 \mathrm{~m})$ on maps of the site carried by the divers. Additional, partial censuses were made at haphazard intervals during the experiment and were successful in locating a handful of tagged gobies missed during the detailed censuses.

At intervals during the experiment, observations were made on the behavior of gobies on each plot. Focal tagged individuals were haphazardly selected and their feeding rate (bites per $5 \mathrm{~min}$ ), aggressive interactions with conspecifics (encounters per $5 \mathrm{~min}$ ) and mean distance from a refuge (in centimeters) were recorded over a 5 -min period. We made a total of $9-10$ observations per plot $(n=171)$, using at least three 
different gobies on each plot $(n=77)$. We also censused piscivorous fishes of the species and sizes that were confirmed predators of gobies (listed in Study species and sites). Each plot was censused for predators on five dates spread over the experiment. Most of the predators had home ranges similar in size to the experimental plots or larger, so the resulting densities are an index of predator visitation.

Gobies were assumed to have died if they disappeared and were not relocated elsewhere. Prior tagrecapture studies of $>4000$ gobies show that goby mortality is well described by an exponential function (Finley and Forrester 2003; G. E. Forrester and M. A. Steele, unpublished data), so daily mortality was calculated as (In (initial number on plot) - In (final number $) \div$ duration of the experiment in days. Gobies were assumed to have emigrated from their plots if they relocated their home range outside their plot during the experiment and then survived to its end. The daily emigration rate was calculated as (n (initial number on plot) - ln (number that emigrated) $) \div$ duration of the experiment in days.

\section{Experiment 2: Does shelter limitation cause density dependence across a range of spatial scales?}

To test if the strength of density dependence was scale dependent, we manipulated density within replicate plots of varying size. We used a three-factor ANCOVA design for this experiment. The first factor was plot size, which we manipulated as a categorical factor with three levels: small $\left(4 \mathrm{~m}^{2}\right)$, medium $\left(16 \mathrm{~m}^{2}\right)$, and large $\left(64 \mathrm{~m}^{2}\right)$. The number of replicate plots differed among plot sizes (small $n=14$, medium $n=6$, large $n=5$ ), because larger plots contained more gobies, so we were more confident in the mortality and migration estimates from them. The second factor was goby density, which was manipulated as a continuous covariate. The range of densities used at each plot size varied (small plots $=0.75-5.50$ gobies $/ \mathrm{m}^{2}$, medium plots $=$ $1.37-4.06$ gobies $/ \mathrm{m}^{2}$, large plots $0.31-3.08$ gobies $/ \mathrm{m}^{2}$ ) to mirror the fact that the range of densities measured naturally changes with plot size. The third factor was refuge density, which was not manipulated and was estimated from the percentage cover of hard substratum in the plot (see Quantifying refuge use in bridled gobies). The estimated refuges density (number per square meter) in plots was treated as a categorical factor with three levels that correspond roughly to the natural distribution of refuges at the site: low (1.0-3.2 refuges/ $\left.\mathrm{m}^{2}\right)$, medium $\left(3.2-4.5\right.$ refuges $\left./ \mathrm{m}^{2}\right)$, and high $(4.5-9.1$ refuges $/ \mathrm{m}^{2}$ ).

Although plots of all three sizes varied in refuge density, because we did not manipulate refuges, we did not have all possible combinations of plot size and refuge abundance. Consequently, we could not test the complete ANCOVA model that included all possible interactions between the effects of plot size, refuge abundance, and goby density. (The three-way inter- action and the two-way interaction between plot size and refuge abundance could not be tested.) We were, however, able to test the remaining two-way interactions between goby density and refuge abundance, and between density and plot size. Testing these latter two interactions was our main goal. First, we wished to test if density dependence was reduced or eliminated where refuges are abundant, which would be indicated by a significant density $\times$ refuges interaction (due to a reduced slope of the relationship between mortality and density at high refuge density). Second, we wished to test if density dependence was scale dependent, which would be indicated in the ANCOVA by a significant interaction between the effect of density and plot size.

This experiment was conducted at Rainbow Reef, near Lee Stocking Island. Many of the procedures were the same as those described previously for Experiment 1 , so we focus our description on points of departure from those methods. As before, experimental plots were simply areas marked at the corners within the site and separated from other plots by 4-6 $\mathrm{m}$. Small plots were square $(2 \times 2 \mathrm{~m})$, as were medium plots $(4 \times 4$ $\mathrm{m})$, whereas large plots were rectangular $(4 \times 16 \mathrm{~m})$ because the site was narrow in places. Goby density was adjusted to the desired level using a mix of resident and transplanted gobies (residents $=27-71 \%$ of all gobies), except that on two of the large plots we did not have time to transplant gobies so we used only the residents and did not manipulate density. Transplanted fish were captured farther away from the experimental site $(2-4 \mathrm{~km})$ than was the case in Experiment 1. After capture, they were held in running seawater at the laboratory for 2-24 h before being tagged, then taken to the experimental site and released onto the appropriate plot by divers.

Like the experiment on Guana Island, this one took several days to set up. Residents were tagged anywhere between 3 and 20 days before the start of the experiment, and transplanted fish were stocked 2-14 days before the start of the experiment. As before, the start date was staggered, in this case over 18 days (from 27 August to 14 September 1998). Once the experiment began, divers censused the plots at intervals. Large plots were censused on day $0,1,4,11$, and finally on day 43. Medium plots were censused on days $0,1,4$, and the final count was on day 37 . Small plots were censused only at the first (day 0) and final day (day 34). Census procedures and methods of calculating mortality and emigration were identical to those used in Experiment 1. As in Experiment 1, we initially analyzed data on residents and transplants separately to test whether transplanted gobies died or emigrated at different rates from residents.

\section{Analyses}

Prior to testing our hypotheses using ANCOVA and $t$ tests, we checked the assumptions of normality and homogeneity of variance (following Sokal and Rohlf 


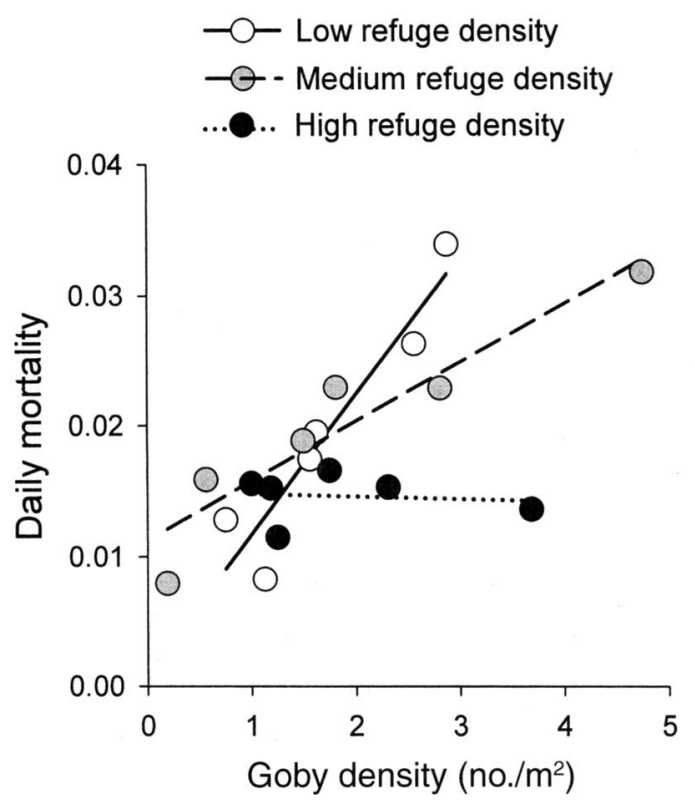

FIG. 1. Relationship between goby density and mortality on replicate plots of reef in Experiment 1. The density of refuges from predation (crevices at the reef/sand interface) was adjusted to one of three levels: low, medium, and high.

1995), and these assumptions were met in all cases. We used post hoc pooling procedures to sequentially remove nonsignificant interaction terms from ANCOVA models, starting with the highest order interactions, using removal criteria described by Winer and coworkers (1991).

\section{RESULTS}

Experiment 1: Is density dependence caused by a shortage of refuges?

Our initial ANCOVA tested for effects of refuge density, goby density, and the interaction between these factors on goby mortality. The interaction was significant $\left(F_{2,12}=14.3, P=0.001\right)$ because gobies suffered progressively higher mortality at high densities in areas where refuge availability was low or intermediate, but not where shelter was abundant (Fig. 1). A shortage of refuges is thus a cause of density dependence because the negative effect of crowding was ameliorated when we increased access to refuges.

The overall mortality of transplanted gobies (mean $\pm 1 \mathrm{SE}=0.024 \pm 0.004)$ was slightly greater than that of residents $(0.016 \pm 0.001)$, and this difference was significant (paired $t$ test, df $=17, t=2.31, P=0.041$ ). We therefore performed separate ANCOVAs on the transplanted and resident gobies, to assess whether the treatments affected the two groups of fish differently. The mortality of both transplants and residents followed the same pattern as that of all fish combined: the intensity of density dependence increased as refuges became scarcer. For transplants, there was a significant interaction between the effects of refuge den- sity and goby density $\left(F_{2,12}=17.3, P=0.003\right)$, and the slopes for each refuge treatment were as follows: high $=-0.0002$, medium $=0.0045$, low $=0.011$. Resident gobies showed the same trend of increasing intensity of density dependence as refuges became scarcer (slopes for each refuge treatment were as follows: high $=0.0015$, medium $=0.0034$, low $=$ 0.0039). For residents, however, the interaction between refuge density and goby density was not significant $\left(F_{2,12}=0.75, P=0.496\right)$ and there was a significant effect of density $\left(F_{1,12}=9.60, P=0.009\right)$. The simplest explanation for the failure to detect a refuge $X$ density interaction in residents is that the mortality estimates were more influenced by random error when based on fewer fish. Overall, the results indicate that gobies suffered effects of refuge shortage at high density.

Few gobies relocated their home range during the experiment (the emigration rate was roughly $10 \%$ of the mortality rate) and there were too few emigrants to assess the emigration rates of resident and transplanted gobies separately. The ANCOVA on total emigration revealed no interactive effect of refuges and density $\left(F_{2,12}=1.08, P=0.372\right)$, but gobies were more likely to leave plots occupied by many rather than few conspecifics (goby density: $F_{1,14}=5.41, P=0.036$ ), and their decision to leave was unaffected by access to refuges (refuge density: $F_{2,14}=0.76, P=0.485$ ).

Neither the feeding rate of gobies nor their mean proximity to shelter was influenced by the experimental treatments. In neither case was there a significant interactive effect of goby density and refuge abundance $\left(F_{1,2}<0.14, P>0.874\right)$, nor was there an influence of either factor separately $\left(F_{1 \text { or } 2,14}<1.65, P>0.220\right)$. There was, however, a significant interactive effect of goby density and refuge density on the per capita rate of aggressive encounters among the gobies $\left(F_{1,2}=5.22\right.$, $P=0.023$ ). At all levels of refuge density, aggression increased with crowding; the interaction occurred because the extent to which aggression increased with density was more pronounced in plots with few refuges than where refuges were at medium or high density (slopes for each refuge treatment were as follows: low $=0.326$, medium $=0.041$, high $=0.073$ ). Despite the fact that transplanted gobies may have suffered more severe effects of refuge shortage than residents, the behavior of the two groups of fish was similar. Transplanted gobies engaged in similar numbers of aggressive interactions with conspecifics (mean $\pm 1 \mathrm{SE}$ ) as residents (transplants $=0.58 \pm 0.15$ encounters $/ 5 \mathrm{~min}$, residents $=0.47 \pm 0.11$ encounters $/ 5 \mathrm{~min} ; t_{124}=0.54$, $P=0.591$ ). Transplanted gobies also did not forage significantly farther from refuges (distance in $\mathrm{cm}$ ) than their resident neighbors (transplants $=15.2 \pm 1.9 \mathrm{~cm}$, residents $\left.=13.0 \pm 1.0 \mathrm{~cm} ; t_{124}=1.04, P=0.300\right)$ nor did they feed at lower rates (bites per $5 \mathrm{~min}$ ): transplants $=3.71 \pm 0.46 \mathrm{bites} / 5 \mathrm{~min}$, residents $=3.15 \pm$ $0.26 \mathrm{bites} / 5 \mathrm{~min} ; t_{124}=1.04, P=0.300$ ). 
Density of refuges

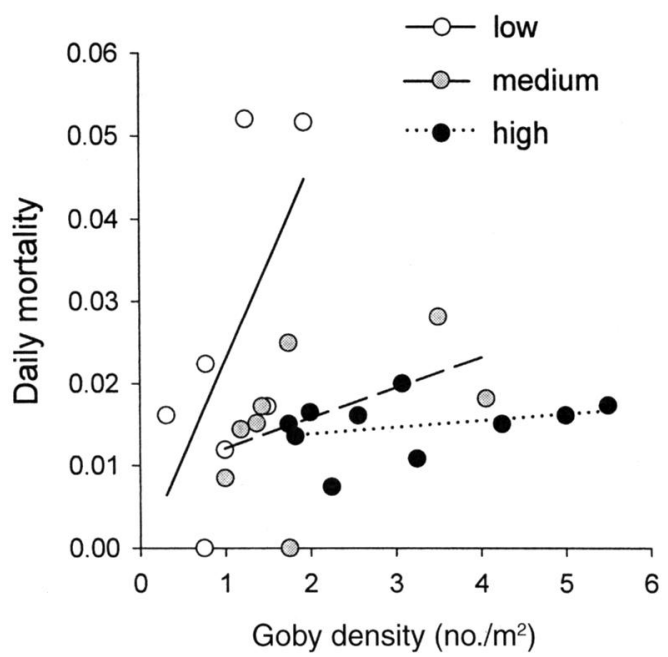

Plot size

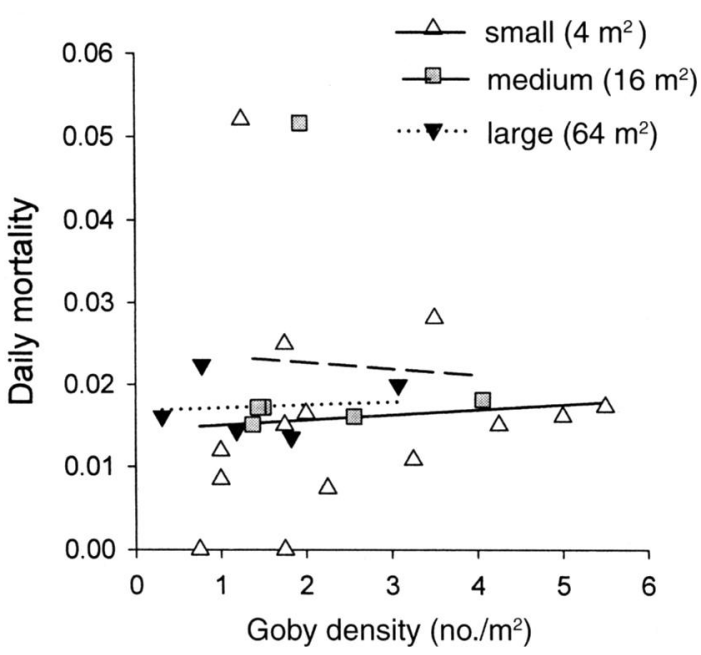

FIG. 2. Relationships between goby density and mortality on replicate plots of reef in Experiment 2. Experimental plots varied in size and also varied naturally in the density of refuges from predation (crevices at the reef/sand interface). Relationships between mortality and density are shown for plots grouped according to plot size (right) and shelter (left).

On average, we saw $1.78 \pm 0.15$ goby predators on a plot on each visit. The predators observed most commonly on the plots (mean number seen per plot per visit $\pm 1 \mathrm{SE})$ were lizardfish $(0.64 \pm 0.09$ fish $)$, followed by hamlets $(0.41 \pm 0.06 \mathrm{fish})$, graysbys $(0.37$ $\pm 0.07 \mathrm{fish})$ and red hinds $(0.27 \pm 0.05$ fish $)$. For none of these predators individually, nor all combined, was their standing density correlated with the density of gobies or the number of refuges in the plot $(n=18, r$ always $<0.19$, Bonferroni-corrected $P$ always $>0.91$ ).

\section{Experiment 2: Does shelter limitation cause density dependence across a range of spatial scales?}

Our ANCOVA tested for effects of plot size, goby density, and refuge density on the mortality of gobies. For reasons described earlier, the ANCOVA model included tests for two interactions (plot size $\times$ goby density, and refuge density $\times$ goby density), but other possible interactions were not tested (see Methods: Experiment 1: Is density dependence caused by limited shelter?). The initial analysis on the mortality of all gobies revealed a significant refuge $\times$ density interaction $\left(F_{2,15}=3.77, P=0.047\right)$, but a nonsignificant plot size $\times$ density interaction $\left(F_{2,15}=0.28, P=\right.$ $0.756)$. The intensity of density-dependent mortality was thus not influenced by the size of the experimental plot, but was influenced by refuge density because the intensity of density dependence increased as refuges became scarcer (Fig. 2).

As in Experiment 1, the mortality rate of transplanted gobies $(0.020 \pm 0.004)$ was slightly higher than that of resident gobies $(0.017 \pm 0.002)$, but the difference was not significant (paired $t$ test, df $=24$, $t=0.71, P=0.487$ ). Nonetheless, we repeated the ANCOVA on the transplanted gobies and the resident gobies separately, to assess whether the treatments affected the two groups differently. For both residents and transplants, there remained no detectable influence of plot size on the strength of density dependence (plot size $\times$ density interaction for residents: $F_{2,15}=$ $0.43, P=0.657$, for transplants: $F_{2,13}=1.19, P=$ $0.351)$. Even though refuge availability had a significant effect on the strength of density dependence when all gobies were pooled, a similar effect was not detectable for either residents or transplants considered separately (refuges $\times$ density interaction: for residents $F_{2,15}=1.54, P=0.256$, for transplants $F_{2,13}=$ $2.08, P=0.187)$. For both resident and transplanted gobies, however, the qualitative pattern of the results was identical to that seen when both groups were pooled (and identical to the patterns seen in Experiment 1). Specifically, the slope of the relationship between density and mortality was lowest in plots with many refuges, intermediate in medium-refuge plots, and greatest in plots with few refuges (regression slopes for residents were: high-density refuges = 0.0019 , medium-density refuges $=0.0043$, low-density refuges $=0.015$; slopes for transients were: highdensity refuges $=-0.0072$, medium-density refuges $=0.0005$, low-density refuges $=0.030)$. Overall, there was thus no suggestion that a shortage of refuges affected only one of the two groups of gobies, and the simplest explanation for failure to detect a refuges $X$ density interaction when residents and transplants were analyzed separately is that the mortality estimates were more influenced by random error when based on fewer fish.

Emigration was very rare during this experiment, as it was in Experiment 1. Only 8 gobies relocated their home range compared to 482 gobies that apparently 
died, so we did not test for treatment effects on emigration.

\section{Discussion}

\section{Predation appears to be the agent of mortality that leads to shelter limitation}

As is true of reef fishes generally, causes of death in gobies can seldom be defined observationally. Consequently, we rely mainly on our collective experimental results on bridled gobies to infer the cause of density-dependent mortality. In 1996, we simultaneously manipulated goby density and predator presence on a set of patch reefs (Forrester and Steele 2000). Gobies used in the 1996 experiment were not tagged, and so we could not separate mortality and emigration. On patch reefs free of predators, gobies showed a low and density-dependent rate of loss, which was almost certainly due to emigration, because we consistently observe low rates of density-dependent emigration from patch reefs (Forrester 1995; G. E. Forrester, unpublished data). On reefs open to predators in the 1996 patch-reef experiment, gobies were lost at a very high and density-dependent rate. Since mortality, and not emigration, always accounted for most losses in other experiments when gobies were tagged and exposed to predators (Forrester 1995; G. E. Forrester and M. A. Steele, unpublished manuscript), predation is almost certainly the cause of high losses from the 1996 reefs open to predators. The combined results of these past experiments, therefore, identify predation as the main agent of mortality in bridled gobies. This conclusion is in accord with our opportunistic field observations of goby deaths, which have all been caused by predators. Density-dependent mortality, then, appears to be caused by increased susceptibility to predation as densities build within areas with few refuges. Various lines of correlative evidence (described in the Introduction) suggest that shelter limitation may be a widespread cause of density-dependent prey mortality in reef fishes, and in some other fish (e.g., Fromentin et al. 2001). But our study is one of few providing strong support for this hypothesis in natural systems (see also Holbrook and Schmitt 2002).

\section{Spatial variation in the intensity of density dependence}

Our findings also clearly indicate that the intensity of density dependence varies spatially according to the local availability of shelter. Spatial variation in the intensity of density dependence has been little studied (Hails and Crawley 1992, Wilson and Osenberg 2002) but has implications for our ability to accurately gauge its strength in heterogeneous habitat. Our results provide an example of a phenomenon dubbed cryptic density dependence by Shima and co-workers (Wilson and Osenberg 2002, Shima and Osenberg 2003). These authors suggest that different classes of habitat can be characterized based on variation in factors that affect their "quality." Because habitat classes differ in quality, each is characterized by a different relationship between density and mortality. In other words, there is not one global relationship between density and mortality, but rather a suite of relationships such that the strength of density dependence differs among habitat classes. For bridled gobies, the local abundance of refuges represents a concrete, measurable facet of habitat quality. We defined classes of habitat that differed in the number of refuges they offered, and showed that the strength of density dependence differed among habitat classes because it was weaker in habitat with plentiful refuges. This situation produces "cryptic" density dependence because failure to identify an underlying factor that influences the strength of density dependence can render it largely undetectable (e.g., Fig. 2) (Wilson and Osenberg 2002, Shima and Osenberg 2003). In observational studies, when density is not manipulated, one reason for the "crypsis" of density dependence is positive covariation between population density and habitat quality (Wilson and Osenberg 2002, Shima and Osenberg 2003). Surveys at our study sites show that the local density of refuges and the density of gobies are, in fact, positively correlated (G. E. Forrester and M. A. Steele, unpublished data). Even when habitat quality does not covary with density, failure to identify the underlying factor influencing the strength of density dependence can still make it difficult to detect. In Experiment 2, our manipulation of density caused refuge availability and goby density to be uncorrelated. Replicate plots, nevertheless, varied naturally in refuge abundance, and this spatial heterogeneity in refuge abundance caused a high degree of scatter in the relationship between density and mortality (Fig. 2). This "density-vagueness" (Strong 1986) was interpretable only when we accounted for spatial heterogeneity in refuge abundance by dividing plots into categories according to the number of refuges they provided (Fig. 2).

Most previous manipulations of fish density on small patch reefs have not considered how the level of refuge abundance, or other habitat features, might influence the strength of density dependence measured (Wilson and Osenberg 2002). In fact, previous experiments have often been done on patch reefs deliberately chosen or constructed to provide homogeneous units of habitat (Jones 1991). One of our previous manipulations of goby density on small patch reefs at Guana Island illustrates this point well. The patches constructed for this 1995 experiment were censused for goby refuges, and refuge density spanned a much narrower range (5.5-7.3 crevices $\left./ \mathrm{m}^{2}\right)$ than observed in natural habitat (see Methods: Quantifying refuge use in bridled gobies). Without information on natural levels and variation in habitat quality, it would be impossible to determine whether this experiment, and others like it, 
have accurately measured the strength of density dependence in nature.

\section{Refuge use and the spatial scaling of density dependence}

The spatial extent of our experiments had no detectable effect on the relationship between goby density and mortality. We suggest two reasons for the consistency of density dependence across spatial scales. The first concerns the nature of refuge use in bridled gobies. The common formulation describing how generalist predators inflict density-dependent mortality considers the situation where prey have a "fixed number" refuge from attack (described in Crawley 1992). The prey population suffers little or no predation at low densities because all of the prey are safe within a refuge. Above a threshold density, some prey must live outside of refuges and so are vulnerable to predation. Some prey species appear to use refuges in this way (Connell 1970, Butler and Herrnkind 1997), including species of reef fish where each individual permanently occupies a single shelter and individuals lacking a shelter are unlikely to survive (Buchheim and Hixon 1992). The nature of refuge use in gobies (described earlier in Methods: Study species and sites) is, however, unlikely to give rise to a fixed number refuge. Gobies retreat to refuges only when threatened, and only for a short time. Because each goby typically has access to several refuges, and most refuges can serve more than one goby, there is unlikely to be a sudden increase in vulnerability once goby densities exceed a threshold set by refuge density. Instead, we expect that vulnerability of any goby to predation will be a progressively increasing function of the number of other gobies in the vicinity and will decline steadily as local refuge density increases. The precise dependence of goby mortality on the number of refuges present is certainly complicated. Nonetheless, we hypothesize that density dependence was consistent in strength across spatial scales because, at all plot sizes, access to refuges was reasonably approximated simply as the number of fish in the plot relative to the number of refuges. We predict that loosely similar relationships between prey density, refuge availability, and vulnerability to predators will occur in other systems where progressive increases in prey safety accrue as the architectural complexity of the habitat increases. Examples include sea grass beds (Hovel and Lipcius 2001), macrophytes (Werner et al. 1983, Babbitt and Tanner 1998), macroalgae (Moksnes et al. 1998, Anderson 2001) and branching corals (Beukers and Jones 1998).

A second factor contributing to the consistent strength of density dependence across spatial scales was the specific mechanism by which predators inflicted density-dependent mortality. Since both gobies and their predators have demographically open populations, a numerical response (Solomon 1949) by predators was not possible. Due to the short duration of the study, a developmental response (Murdoch 1971) by predators also was not possible. Goby predators, considered as a group, also showed no tendency to concentrate their foraging activity in dense prey patches (an aggregative response; Hassell and May 1974). By process of elimination, predators must have caused density-dependent goby mortality simply though a disproportionate increase in capture rate with prey density (a type III functional response; Holling 1959). The lack of spatial association between the densities of piscivorous reef fishes and gobies at the scales we studied probably contributed to the consistency in density dependence across spatial scales. Spatial associations (or lack thereof) between predator and prey density will, however, be species and context specific. In some situations, the density of predatory fish does covary with prey density (Stewart and Jones 2001), and an aggregative response is the putative cause of density-dependent mortality in other reef fishes (Hixon and Carr 1997, Anderson 2001). Our ability to assess the nature and effects of density-dependent interactions on population dynamics should thus be improved by work that identifies the underlying biological interactions causing density-dependent mortality, and characterizes the spatial and temporal domains at which those interactions operate.

\section{ACKNOWLEDGMENTS}

Many thanks to all of those who helped us with field work, including Danielle Ameen, Scott Bull, Bryn Evans, Ian Greenspan, Liz Kintzing, Linda O’Bryan, Rachel Finley, Amy Karpenske, Jenna Krug, Jameal Samhouri, Jake Sausman, Julie Standish, Jeff Schinske, Eric Tynes, Jessica Tallman, Chau Tran, Denise Weisman, and Clare Wormald. We are also grateful for the excellent logistical support provided by Lianna Jarecki and the staffs at the Caribbean Marine Research Center (CMRC) and Guana Island. Financial support was provided by the National Science Foundation (OCE 9618011, 0096061, and 0222087), NOAA-NURP (CMRC01-NRME-01-02A), the Perry Foundation, the Guana Island Wildlife Sanctuary, and the Falconwood Corporation. Views expressed herein are those of the authors and do not necessarily reflect those of CMRC or NOAA. This is contribution number CMRC-01-NRME-01-3C from CMRC.

\section{Literature Cited}

Anderson, T. W. 2001. Predator responses, prey refuges, and density-dependent mortality of a marine fish. Ecology 82: 245-257.

Auerbach, M., E. Connor, and S. Mopper. 1995. Minor miners and major minors: population dynamics of leaf-mining insects. Pages 83-110 in N. Cappuccino and P. Price, editors. Population dynamics: new approaches and synthesis. Academic Press, San Diego, California, USA.

Ault, T. R., and C. R. Johnson. 1998. Relationships between habitat and recruitment of three species of damselfish (Pomacentridae) at Heron Reef, Great Barrier Reef. Journal of Experimental Marine Biology and Ecology 223:145-166.

Babbitt, K. J., and G. W. Tanner. 1998. Effects of cover and predator size on survival and development of Rana utricularia tadpoles. Oecologia 114:258-262.

Begon, M., M. Mortimer, and D. J. Thompson. 1996. Population ecology: a unified study of animals and plants. Third edition. Blackwell Scientific, Oxford, UK. 
Bertolo, A., G. LaCroix, and F. Lescher-Moutoué. 1999. Scaling food chains in aquatic mesocosms: do the effects of depth override the effects of planktivory? Oecologia 121: $55-65$.

Beukers, J. S., and G. P. Jones. 1998. Habitat complexity modifies the impact of piscivores on a coral reef fish population. Oecologia 114:50-59.

Buchheim, J. R., and M. A. Hixon. 1992. Competition for shelter holes in the coral-reef fish Acanthemblemaria spinoza Metzelaar. Journal of Experimental Marine Biology and Ecology 164:45-54.

Butler, M. J. I., and W. F. Herrnkind. 1997. A test of recruitment limitation and the potential for artificial enhancement of spiny lobster (Panulirus argus) populations in Florida. Canadian Journal of Fisheries and Aquatic Sciences 54:452-463.

Caley, M. J., M. H. Carr, M. A. Hixon, T. P. Hughes, G. P. Jones, and B. A. Menge. 1996. Recruitment and the population dynamics of open marine populations. Annual Review of Ecology and Systematics 27:477-500.

Caley, M. J., and J. St John. 1996. Refuge availability structures assemblages of tropical reef fishes. Journal of Animal Ecology 65:414-428.

Cappuccino, N. 1992. The nature of population stability in Eurosta solidaginis, a nonoutbreaking herbivore of goldenrod. Ecology 73:1792-1801.

Cappuccino, N., and P. W. Price. 1995. Population dynamics: new approaches and synthesis. Academic Press, San Diego, California, USA.

$\rightarrow$ Carr, M. H., T. M. Anderson, and M. A. Hixon. 2002. Biodiversity, population regulation, and the stability of coralreef fish communities. Proceedings of the National Academy of Sciences (USA) 99:11241-11245.

Carr, M. H., and M. A. Hixon. 1995. Predation effects on early post-settlement survivorship of coral-reef fishes. Marine Ecology Progress Series 124:31-42.

Connell, J. H. 1970. A predator prey system in the marine intertidal region. I. Balanus glandula and several predatory species of Thais. Ecological Monographs 40:49-78.

Connell, S. D. 1997. The relationship between large predatory fish and recruitment and mortality of juvenile coral reef-fish on artificial reefs. Journal of Experimental Marine Biology and Ecology 209:261-278.

Crawley, M. J. 1992. Natural enemies: the population biology of predators, parasites, and diseases. Blackwell Scientific, Oxford, UK.

Doherty, P. J. 1991. Spatial and temporal patterns of recruitment. Pages 261-293 in P. F. Sale, editor. The ecology of fishes on coral reefs. Academic Press, San Diego, California, USA.

Doherty, P. J. 2002. Variable replenishment and the dynamics of reef fish populations. Pages 327-355 in P. F. Sale, editor. Coral reef fishes: dynamics and diversity in a complex ecosystem. Academic Press, San Diego, California, USA.

Finley, R. J., and G. E. Forrester. 2003. Impact of ectoparasites on the demography of a small reef fish. Marine Ecology Progress Series 248:305-309.

Force, D. C., and D. J. Moriarty. 1988. Effects of patch scale on density-dependence and species-dependence in two host-parasitoid systems. Oecologia 76:439-444.

Forrester, G. E. 1990. Factors influencing the juvenile demography of a coral reef fish. Ecology 71:1666-1681.

Forrester, G. E. 1995. Strong density-dependent survival and recruitment regulate the abundance of a coral reef fish. Oecologia 103:275-282.

Forrester, G. E., and M. A. Steele. 2000. Variation in the presence and cause of density-dependent mortality in three species of reef fishes. Ecology 81:2416-2427.

Freeman, B., and D. Smith. 1990. Variation of density-dependence with spatial scale in the leaf mining fly Liriomyza commelinae (Diptera: Agromyzidae). Ecological Entomology 15:265-274.

Fromentin, J. M., R. A. Myers, O. N. Bjornstad, N. C. Stenseth, J. Gjosaeter, and H. Christie. 2001. Effects of densitydependent and stochastic processes on the regulation of cod populations. Ecology 82:567-579.

Hails, R. S., and M. J. Crawley. 1992. Spatial density dependence in populations of a cynipid gall-former Andricus Quercuscalicis. Journal of Animal Ecology 61:567-583.

Harrison, S., and N. Cappuccino. 1995. Using density-manipulation experiments to study population regulation. Pages 131-172 in N. Cappuccino and P. W. Price, editors. Population dynamics: new approaches and synthesis. Academic Press, San Diego, California, USA.

Hassell, M. P., and R. M. May. 1974. Aggregation of predators and insect parasites and its effect on stability. Journal of Animal Ecology 43:567-594.

Helfman, G. S. 1993. Fish behaviour by day, night and twilight. Pages 56-71 in T. J. Pitcher, editor. Behaviour of teleost fishes. Chapman and Hall, London, UK.

Hixon, M. A., and M. H. Carr. 1997. Synergistic predation, density dependence, and population regulation in marine fish. Science 277:946-949.

Hixon, M. A., and M. S. Webster. 2002. Density dependence in marine fishes: coral-reef populations as model systems. Pages 303-325 in P. F. Sale, editor. Coral reef fishes: dynamics and diversity in a complex ecosystem. Academic Press, San Diego, California, USA.

Holbrook, S. J., G. E. Forrester, and R. J. Schmitt. 2000. Spatial patterns in abundance of a damselfish reflect availability of suitable habitat. Oecologia 122:109-120.

Holbrook, S. J., and R. J. Schmitt. 2002. Competition for shelter space causes density-dependent predation mortality in damselfishes. Ecology 83:2855-2868.

Holling, C. S. 1959. The components of predation as revealed by a study of small-mammal predation of the European pine sawfly. Canadian Entomologist 91:293-320.

Hopper, K. R., J. E. Powell, and E. G. King. 1991. Spatial density dependence in parasitism of Heliothis virescens (Lepidoptera: Noctuidae) by Microplitis croceipes (Hymenoptera: Braconidae) in the field. Environmental Entomology 20:292-302.

Hovel, K. A., and R. N. Lipcius. 2001. Habitat fragmentation in a seagrass landscape: patch size and complexity control blue crab survival. Ecology 82:1814-1829.

Jones, G. P. 1991. Postrecruitment processes in the ecology of coral reef fish populations: a multifactorial perspective. Pages 293-328 in P. F. Sale, editor. The ecology of fishes on coral reefs. Academic Press, London, UK.

Karieva, P., and M. Anderson. 1986. Spatial aspects of species interactions: the wedding of models and experiments. Pages 35-50 in A. Hastings, editor. Community ecology: lecture notes in biomathematics. Springer-Verlag, Berlin, Germany.

Kawat, M. 1997. Exploitative competition and ecologically effective abundance. Ecological Modelling 94:125-137.

Lynch, L. D., R. G. Bowers, M. Begon, and D. J. Thompson. 1998. A dynamic refuge model and population regulation by insect parasitoids. Journal of Animal Ecology 67:270279.

Malone, J. C., G. E. Forrester, and M. A. Steele. 1999. Effects of subcutaneous microtags on the growth, survival, and vulnerability to predation of small reef fishes. Journal of Experimental Marine Biology and Ecology 237:243-253.

Moksnes, P.-O., L. Pihl, and J. Van Montfrans. 1998. Predation on postlarvae and juveniles of the shore crab Carcinus maenas: importance of shelter, size and cannibalism. Marine Ecology Progress Series 166:211-225.

Murdoch, W. 1971. The developmental response of predators to changes in prey density. Ecology 52:132-137. 
Murdoch, W., and A. Oaten. 1975. Predation and population stability. Advances in Ecological Research 9:1-132.

Osenberg, C. W., C. M. St Mary, R. J. Schmitt, S. J. Holbrook, P. Chesson, and B. Byrne. 2002. Rethinking ecological inference: density dependence in reef fishes. Ecology Letters 5:715-721.

Ray, C., and A. Hastings. 1996. Density dependence: are we searching at the wrong spatial scale? Journal of Animal Ecology 65:556-566.

Rogers, C. S., T. H. Sucharek, and F. A. Pecora. 1982. Effects of hurricanes David and Frederic (1979) in shallow Acropora palmata communities: St. Croix, U.S. Virgin Islands. Bulletin of Marine Science 32:532-548.

Rothman, L. D., and D. C. Darling. 1990. Parasitoids of the goldenrod gall moth: effects of scale on spatial density dependence. Oecologia 83:1-6.

Shima, J. S., and C. W. Osenberg. 2003. Cryptic density dependence: effects of covariation between density and site quality in reef fish. Ecology 84:46-52.

Sokal, R. R., and F. J. Rohlf. 1995. Biometry: the principles and practice of statistics in biological research. Third edition. W. H. Freeman, New York, New York, USA.

Solomon, M. E. 1949. The natural control of animal populations. Journal of Animal Ecology 18:1-35.

Sponaugle, S., and R. K. Cowen. 1994. Larval durations and recruitment patterns of two Caribbean gobies (Gobiidae) contrasting early life histories in demersal spawners. Marine Biology 120:133-143.

Steele, M. A. 1997. The relative importance of processes affecting recruitment of two temperate reef fishes. Ecology 78:129-145.
Steele, M. A. 1999. Effects of shelter and predators on reef fishes. Journal of Experimental Marine Biology and Ecology 233:65-79.

Steele, M. A., and G. E. Forrester. 2002. Early postsettlement predation on three reef fishes: effects on spatial patterns of recruitment. Ecology 83:1076-1091.

Stewart, B. D., and G. P. Jones. 2001. Associations between the abundance of piscivorous fishes and their prey on coral reefs: implications for prey-fish mortality. Marine Biology 138:383-397.

Strong, D. R. 1986. Density-vague population change. Trends in Ecology and Evolution 1:39-42.

Werner, E. E., J. F. Gilliam, D. J. Hall, and G. G. Mittlebach. 1983. An experimental test of the effects of predation risk on habitat use in fish. Ecology 64:1540-1548.

Whitlatch, R. B., A. H. Hines, S. F. Thrush, J. E. Hewitt, and V. Cummings. 1997. Benthic faunal responses to variations in patch density and patch size of a suspension-feeding bivalve. Journal of Experimental Marine Biology and Ecology 216:171-189.

Wiens, J. A. 1989. Spatial scaling in ecology. Functional Ecology 3:383-397.

Wilson, J., and C. W. Osenberg. 2002. Experimental and observational patterns of density-dependent settlement and survival in the marine fish Gobiosoma. Oecologia 130:205215.

Winer, B. J., D. R. Brown, and K. M. Michels. 1991. Statistical principles in experimental design. McGraw-Hill, New York, New York, USA. 